\title{
Caracterización general y evolución de la personalidad en la primera infancia
}

\author{
General characterization and personality evolution in early childhood \\ Caracterização geral e evolução da personalidade na primeira infância
}

ARTÍCULO DE INVESTIGACIÓN

\author{
Charly Marlene Valarezo Encalada \\ charly.valarezo@unae.edu.ec \\ ORCID: 0000-0002-6540-8395
}

Universidad Nacional de Educación, Ecuador

Sonia Zhadira Celi Rojas

sonia.celi@unl.edu.ec

ORCID: 0000 - 0001-6513-2168

Universidad Nacional de Loja, Ecuador

\author{
Diana Belén Rodríguez Guerrero \\ diana.b.rodriguez@unl.edu.ec \\ ORCID: 0000 - 0003-1155-8395
}

Universidad Nacional de Loja, Ecuador
Viviana Catherine Sánchez Gahona viviana.sanchez@unl.edu.ec
ORCID: 0000 - 0001-5662-6115

Universidad Nacional de Loja, Ecuador

Recibido 11 de octubre 2020 | Arbitrado y aceptado 28 de noviembre 2020 | Publicado en diciembre 2020

\section{RESUMEN}

Este artículo destaca el análisis de las diferentes posiciones teóricas relacionadas al desarrollo de la personalidad, las principales características evolutivas entorno a la misma y su influencia en la vida socio afectivo, sin dejar de lado la influencia que tiene el contexto familiar. El objetivo que demanda interés es caracterizar la evolución socioafectiva del niño de 0 a 6 años como un componente básico de la personalidad. Se utilizó el método descriptivo, y el analítico sintético para el análisis de las unidades de estudio. Se concluyó que la personalidad será el resultado del temperamento y las acciones socio educativas que reciba de los adultos más cercanos que lo rodean, su influencia favorece o no su desarrollo sano. De tal manera que, las conductas se irán consolidando en función de las reacciones o estímulos que la familia manifieste ante su comportamiento y de las diferentes experiencias que vaya acumulando.

Palabras clave: Desarrollo de la personalidad; primera infancia; socio afectividad, familia

\section{ABSTRACT}

This article highlights the analysis of the different theoretical positions related to the development of the personality, the main evolutionary characteristics around it and its influence on socio-affective life, without neglecting the influence of the family context. The objective that demands interest is to characterize the socioaffective evolution of the child from 0 to 6 years old as a basic component of the personality. The descriptive method and the synthetic analytical one were used for the analysis of the study units. It was concluded that the personality will be the result of the temperament and the socio-educational actions that it receives from the closest adults that surround it, its influence favors or not its healthy development. In such a way that, the behaviors will be consolidated according to the reactions or stimuli that the family shows to their behavior and the different experiences that they accumulate.

Key words: Personality development; early childhood; social affectivity, family 


\section{RESUMO}

Este artigo destaca a análise das diferentes posições teóricas relacionadas ao desenvolvimento da personalidade, as principais características evolutivas ao seu redor e sua influência na vida socioafetiva, sem descurar a influência do contexto familiar. 0 objetivo que demanda interesse é caracterizar a evolução socioafetiva da criança de 0 a 6 anos como componente básico da personalidade. 0 método descritivo e o analítico sintético foram utilizados para a análise das unidades de estudo. Concluiu-se que a personalidade será fruto do temperamento e das ações socioeducativas que recebe dos adultos mais próximos que a cercam, sua influência favorece ou não o seu desenvolvimento saudável. De forma que, os comportamentos serão consolidados de acordo com as reações ou estímulos que a família apresenta ao seu comportamento e as diferentes experiências que acumula.

Palavras-Chave: Desenvolvimento da personalidade; primeira infância; afetividade social, família

\section{INTRODUCCIÓN}

El anhelo de estudiar la personalidad, se remonta a épocas pasadas y se mantiene en intereses actuales; se habla continuamente de la necesidad de educar la personalidad del Eniño. Sin embargo, esto implica un elevado ideal, las continuas variantes en las condiciones de vida a las que los infantes están expuestos, afectan principalmente la formación de ésta. En consecuencia, esta investigación resalta la caracterización de la evolución socio afectiva del niño de 0 a 6 años como un componente básico de la personalidad. Se aborda entonces, una explicación desde los factores que influyen en el desarrollo de la misma a partir de las edades tempranas.

El desarrollo de la personalidad obedece a varios factores, uno de éstos puede estar direccionado en el momento de la concepción cuando las células del niño reciben información. Proceso que se va solidificando en el nacimiento cuando entra en contacto con el entorno y se consolida con las bases socio afectivas que recibe de su contexto inmediato. En definitiva, el individuo se ve influenciado por factores endógenos y exógenos.

El contexto familiar es primordial para el desarrollo, aquí se forjan los primeros vínculos afectivos que sentarán las bases para una vida emocional equilibrada. Además, es la mediadora de las experiencias de aprendizaje del niño, es responsable de la seguridad emocional y de la construcción de sus creencias, valores, fortalecimiento de su autoestima, apoyando el desarrollo del sistema de pensamiento y toma de decisiones. La familia también interviene en el proceso de educación del niño inicialmente con el ejemplo, con las vivencias propias dentro del hogar y luego en la inserción al sistema educativo formal.

Otro de los factores influyentes en la edad temprana es el medio social, ya que permite afianzar el aprendizaje y mejorar las áreas de desarrollo. Sumando, es un aspecto determinante para que una persona pueda desarrollarse de manera íntegra y correcta en la sociedad (Quezada, 2018). Por lo tanto, la socialización es uno de los elementos significativos porque le permite al niño adquirir estrategias y habilidades sociales en su entorno familiar para proyectarlas en otros contextos. Este hecho permite al sujeto tomar decisiones oportunas y comprender distintos factores característicos de la etapa que atraviesa, para ello será necesario la atención en la primera infancia donde se consolidan las bases de la personalidad.

El estudio del desarrollo de la personalidad es un tema de gran relevancia, porque permite entender cómo es su formación y los factores que la afectan, lo que pone a manifiesto que en tal proceso no existen patrones únicos $\mathrm{y}$ permanentes, además, el análisis de las experiencias tempranas, los procesos de 
identificación con sus figuras parentales, la forma en la que va resolviendo los conflictos propios del desarrollo, permite profundizar y sustentar el presente estudio.

Se analiza las características, factores, teorías y elementos básicos de la personalidad; desarrollo de la personalidad; y el rol de la familia como principal agente de socialización.

\section{MÉTODO}

El trabajo propuesto se enmarca en el nivel de una revisión descriptiva, puesto que "proporciona al lector una puesta al día sobre conceptos útiles en áreas en constante evolución" (Guirao-Goris, Olmedo Salas y Ferrer Ferrandis, 2008, p.6). Y en el campo de la educación inicial tener información de primera mano, brinda al docente la oportunidad de reflexionar sobre los hitos de desarrollo del infante. Específicamente en el tema de estudio, en comprender cómo los vínculos afectivos y sociales van a configurar la base del desarrollo de la personalidad de los niños.

Atendiendo al objetivo de este trabajo (Caracterizar la evolución socio afectiva del niño de 0 a 6 años como un componente básico de la personalidad), se estableció que la categoría de análisis se apoya en las siguientes preguntas de investigación: ¿Qué se conoce sobre el desarrollo de la personalidad en la primera infancia?, ¿A qué hace referencia el desarrollo socio afectivo en la primera infancia? y ¿Cuál es el rol de la familia como principal agente de socialización? Esto permitió profundizar crítica y reflexivamente en el tema de interés.

El proceso de indagación se cimenta en la búsqueda de publicaciones indexadas en bases de datos electrónicos, tales como el buscador académico Google Scholar; la plataforma digital Web of Science; Redalyc; Scielo; y otras bases de datos especializadas en educación. Se utilizaron los siguientes descriptores: "caracterización socio afectiva", "primera infancia", "evolución de la personalidad" y "agentes de socialización". Posterior a la revisión para dar sentido, profundidad y secuencialidad en el abordaje del tema, se definieron cinco aspectos de análisis: características y factores en la formación de la personalidad; diferentes posiciones teóricas; desarrollo de la personalidad en la niñez temprana; elementos básicos de la personalidad; y el rol de la familia como principal agente de socialización.

El período de búsqueda documental se estableció a 10 años, es decir, publicaciones desde 2010 hasta 2020, considerando que en la actualidad los aportes a la comunidad científica se actualizan constantemente. En el proceso de exploración y selección para el análisis cualitativo se revisaron alrededor de 61 artículos, de los cuales fueron considerados 34 para el presente estudio. Se siguió las orientaciones de la declaración PRISMA (Liberati, 2009), cuyo propósito fue garantizar la trasparencia y claridad en la depuración de la información, descartando los artículos que no contenían información suficientemente relacionada con el objetivo de la investigación.

RESULTADOS

La personalidad ha sido desde siempre un objeto de estudio para muchos autores hasta la actualidad, siendo importante señalar la subjetividad conceptual debido a la perspectiva o inclinación teórica de cada autor.

El concepto que se tomará como referente para el presente artículo es: "La personalidad es la estructura dinámica que tiene un individuo en particular; se compone de características psicológicas, conductuales, emocionales y sociales" (Seelbach, 2013, p.9). Reconociendo que el proceso de construcción 
de la personalidad no es un suceso con características puntuales y tampoco se lo puede generalizar a todos los niños de la misma forma, esto responde a un desarrollo evolutivo sistemático.

Es importante señalar, que en la actualidad existe controversia sobre la formación de la personalidad, unos autores consideran que está formada a partir de una base genética y por otra parte ambiental, fenómeno que ocurre en los primeros años de vida; para otros, la personalidad se va formando a través del ciclo vital extendiéndose desde la infancia hasta los treinta años de edad, momento estable del ser humano. Sin embargo, existen autores que aducen que la personalidad es flexible y sigue evolucionando a lo largo de la vida adulta (Caballo, Salazar e Irurtria, 2011).

Al respecto Romero (2002), comenta que "los rasgos de personalidad son el resultado de factores complejos y de múltiples interacciones de elementos genéticos, neuropsicológicos y neuroquímicos con manifestaciones conductuales y manifestaciones sociales" (p.140). Se suma a este criterio Morán, Fínez y Fernández-Abascal (2017), quienes señalan que los rasgos de personalidad son el producto de la interacción de la herencia con el ambiente, mismos que ejercen su influencia en la estructura de la personalidad y en el curso de futuros aprendizajes.

No se puede definir con total seguridad que los elementos citados tengan mayor relevancia uno del otro, ya que desde una mirada biologista, posibilita comprender ciertos criterios, pero por otro lado resulta una limitante para el individuo, la cultura y sociedad. Además, desde la concepción genética no se ha comprobado científicamente que los genes influyen en los componentes afectivos, cognitivos y conductuales de los individuos, aún es tema de estudio y controversia entre muchos autores.
En la formación de la personalidad, Tintaya (2019) cita los siguientes factores:

- Factores genéticos: características innatas con ciertas disposiciones físicas donde se estructura la personalidad.

- Factores ambientales: experiencias vividas que se generan gracias al medio ambiente, con influencia en el desarrollo psicológico.

- Factores de determinación: difiere de los anteriores y se refiere al poder creativo del sujeto, en el que predomina la capacidad de autodeterminación del ser humano, con funciones autorreguladoras. Atiende las necesidades, aspiraciones que dan sentido a su vida, potencializando la misma.

Por su parte, Carrillo Siles (2009) sugiere que en la formación de la personalidad intervienen cuatro tipos de factores. El primero hace referencia a la herencia como una constitución física y una dotación genética. El segundo hace alusión al ambiente, en el que se proporciona significado a los estímulos que provienen del mundo exterior. El tercero son las expresiones de carácter psicológico, en donde se da atención a los aspectos afectivos y sociales y, finalmente, los valores que son inculcados desde etapas tempranas, convirtiéndose en reglas de conducta temprana que determinan el comportamiento.

Para Simkin y Azzollini (2015), siguiendo los postulados de McCrae y Costa (2012), "los individuos reaccionan al ambiente a partir de patrones de pensamientos, sentimientos y conductas consistentes con sus rasgos de personalidad, modelando estructuras psicológicas que guían el comportamiento: hábitos, valores, planes, habilidades, esquemas y relaciones, denominadas características adaptativas" (p.343). En referencia a nuestra población de estudio que es la infancia, y 
apoyados en los estudios científicos, podemos aseverar que la formación de la personalidad se genera a través de una conjugación de elementos que van dando sentido y originalidad a cada ser humano. Por lo tanto, contribuyen a la construcción del temperamento y carácter.

\section{Diferentes posiciones teóricas}

Craig (2009) enumera tres teorías que estudian el desarrollo de la personalidad en la infancia.

Teorías psicodinámicas. El niño experimenta una serie de emociones $y$ sentimientos, positivos y negativos, por ende, la importancia en la adquisición de mecanismos reguladores aceptables y las normas sociales le facultan de medios para expresarse y comportarse siendo consciente entre lo que se desea y lo que puede hacer.

Conforme va creciendo debe ir aprendiendo a superar conflictos acordes con su desarrollo. La necesidad de autonomía, a partir de la relación con las figuras de autoridad, surge de la necesidad de realizar las cosas por sí solo para sentirse competente y exitoso en su entorno físico $\mathrm{y}$ social. El asumir estos retos oportunamente librará al niño de futuros problemas.

Teorías del aprendizaje social: el comportamiento está influenciado por el ambiente y las interacciones sociales en las que el niño obtiene información en relación con los modelos de roles a los que tiene acceso, lo que determina rasgos en la formación de la personalidad, "el repertorio social del preescolar se ve profundamente influido por el juego $\mathrm{y}$ por otras interacciones con los compañeros [...] este sin duda mejora sus habilidades sociales, pues les permite que se ejerciten en sus relaciones con los demás" (p.167). Sin duda el juego en su entorno escolar constituye una influencia predominante en la construcción de su imagen.
Erikson, recogiendo algunas ideas de Freud, propone la necesidad de establecer relaciones sociales del ser humano. De esta manera, mantiene que el desarrollo humano se produce por estadios psicosociales, en donde es vital la vinculación con otras personas. Durante el primer año de vida, el niño necesita desarrollar el sentimiento de confianza basado en el cuidado físico que le es conferido por sus padres, en donde comienza a descubrir su propia identidad $\mathrm{y}$, por lo tanto, sus propios comportamientos. Este primer estadio es fundamental para el desarrollo del segundo. $\mathrm{Si}$ el niño ha experimentado confianza y ha tenido límites seguros y adecuados desarrolla su independencia y autonomía, en efecto podrá reafirmar tales características a lo largo de los siguientes estadios de desarrollo.

Bowlby (1973) por su parte, defiende desde el inicio la necesidad del contacto social que tenemos los individuos desde el nacimiento hasta la vejez. Señala que desde que nacemos tenemos necesidades primarias de socialización y afectividad y que, además, ésta es correspondida adecuadamente por los cuidadores adultos.

Teorías cognitivas. El niño acorde con su evolución física y emocional va adquiriendo el concepto del yo. El desarrollo de su personalidad puede estar influenciado por factores determinantes como las habilidades cognitivas que le permiten asimilar, comprender, descubrir y optar por conductas y autorregularlas de acuerdo con el bagaje de experiencias acumuladas de juicios internos y externos que influyen para crear pautas globales de comportamiento que sean apropiados a su individualidad, familia $\mathrm{y}$ cultura.

De acuerdo a Montaño, Palacios y Gantiva (2009), existe también: 
Teoría fenomenológica de la personalidad. esta teoría tiene como perspectiva fundamental que el ser humano no debe ser comprendido como resultado de conflictos ocultos e inconscientes, debe ser visto desde un enfoque de motivación positiva y que de acuerdo a su evolución logrará un funcionamiento a niveles superiores como agente responsable de sus actos y por lo tanto de las consecuencias producto de estos.

Teoría de los rasgos. Hace referencia al individuo y sus características particulares como son: el temperamento, la adaptación, la labilidad emocional y los valores que le permiten al individuo girar en torno a una característica en particular (Engel, 1996). Raymond Cattel, figura importante en relación a esta teoría propuso una clasificación de los rasgos: a) comunes (propios de todas las personas) contra únicos (son característicos del individuo); b) superficiales (fáciles de observar) contra fuentes (solo pueden ser descubiertos mediante análisis factorial); c) constitucionales (dependen de la herencia), contra moldeados por el ambiente (dependen del entorno); d) los dinámicos (motivan a la persona hacia la meta), contra habilidad (capacidad para alcanzar la meta), contra temperamento (aspectos emocionales de la actividad dirigida hacia la meta) (Aiken, 2003).

Teoría conductual de la personalidad. El enfoque conductual de la personalidad hace énfasis en la especificidad situacional restándole importancia a las manifestaciones internas (Pervin y Jhon, 1998). Skinner determina que la conducta es producto específico del ambiente a través de estímulos que actúan como reforzadores que propiciarán la incidencia conductual.

Siendo que, "la personalidad permite conocer de forma aproximada los motivos que llevan a un individuo a actuar, sentir, pensar y desenvolverse en un medio; está compuesta de características psicológicas, conductuales, emocionales y sociales" (González, 2013, p. 13). Configurándose por dos componentes o factores: el temperamento y el carácter: (González, 2013).

Temperamento: es un componente de la personalidad que determina, de alguna manera, ciertas características de cognición, comportamiento y emociones.

Carácter: son las características de la personalidad que son aprendidas en el medio, por ejemplo, los sentimientos son innatos, es decir, se nace con ellos, pero la manera en cómo se expresan forma parte del carácter. Las normas sociales, los comportamientos y el lenguaje, son solo algunos componentes del carácter que constituyen a la personalidad.

De esta manera "desde el inicio cada uno muestra una personalidad distinta: la mezcla de emociones temperamento, pensamiento y conducta, hace única a cada persona" (Papalia, Feldman, y Martorell, 2012, p.174).

\section{Desarrollo de la personalidad en la niñez temprana}

La construcción continua de la personalidad, más allá de formar al individuo, le establece un patrón de vida inicialmente guiado por los padres durante los primeros años de vida hasta que logre su autonomía, es entonces cuando cada ser humano empieza a escribir su propia historia basado en esos procesos adquiridos inicialmente.

De 0 a 12 meses: En esta etapa existe un factor determinante en el desarrollo del niño que es el apego, la capacidad de aprender, de buscar estímulos, y vínculos afectivos desde el propio nacimiento, y el rol que cumple la lactancia es preponderante, ya que su aporte en seguridad, independencia y estabilidad emocional es indiscutible.

El papel que desempeña el adulto o figura significativa es demandante, sus niveles de 
interpretación sobrepasan barreras, donde, el niño depende en su totalidad para satisfacer sus necesidades básicas tanto fisiológicas como afectivas.

Se inicia una etapa importante de descubrimiento, de a poco aumenta su independencia y las ganas de explorar todo lo que le rodea, es decir, se inicia un despertar sensorial que favorece el desarrollo cognitivo del niño.

De 1 a 3 años: El niño en esta etapa desarrolla un mayor sentido de autocontrol sobre su entorno. El apoyo de los agentes de socialización, en especial de la familia, permite al niño desarrollar la confianza en sí mismo y la autoestima gracias a las manifestaciones de amor y de reconocimiento que colman sus necesidades básicas: afecto, aceptación y seguridad. Los niños que completen con éxito esta etapa se sienten seguros y confiados, mientras que los que no, se quedan con un sentimiento de incapacidad y falta de confianza.

El propósito de una paternidad efectiva se basa en la comunicación, los afectos, la instauración de normas y reglas, establecer límites, la estimulación de cada una de sus dimensiones, brindar apoyo permanente, fomentar el sentido de pertenencia y el acompañamiento permanente.

En determinado momento el desarrollo social y cognitivo permite al niño descubrir diversas aristas de las múltiples inteligencias, por ejemplo, la expresión artística, musical, verbal, inter e intra personal, entre otras, lo que le permite manejar de forma adecuada sus emociones.

De 4 a 6 años: Para Altajeros (2004), en la función familiar como agente de crianza y socialización, permite que los hijos desarrollen un proyecto de vida que abarca todos los ámbitos de su desarrollo. En lo que respecta a la función afectiva, los padres expresan sentimientos, generan autoestima, y seguridad.
Además de la afectividad comparten actividades en el hogar, se fortalece la comunicación abierta y clara, asumen errores y responsabilidades de las decisiones tomadas.

Linares, (2009) afirma que:

Para esta etapa, el desarrollo que se tiene en la niñez les permite tener la capacidad de recordar imágenes y poder codificar sus experiencias en símbolos, por tal motivo es que se recomienda el uso del juego simbólico, ya que «favorece el desarrollo del lenguaje, así como las habilidades cognoscitivas y sociales [...] (p. 27).

Es decir, si se juega en la modalidad simbólica, ya sea de manera grupal o no, el niño siempre aportará la experiencia que ha adquirido, expresando su realidad interna, como se observa, el juego simbólico permite que el niño refleje el sentido propio que tiene acerca de la realidad.

Para el niño resulta necesario adaptarse a un mundo que es inminentemente social y, siendo el mismo altamente complejo, son diversas las posibilidades que permite el estímulo sensorial, cognitivo y afectivo adecuado, ya que entre tantos aportes encontramos que le ayuda a crear un mundo propio en el que se va familiarizando de a poco con reglas e intereses que le resultan desconocidos.

\section{Elementos básicos de la personalidad}

Desde la misma concepción se va construyendo la vida afectiva del ser humano, el primer año de vida es la etapa donde el afecto es totalmente decisivo para el adecuado desarrollo de la personalidad, pero en los años subsecuentes también.

Cuando hablamos de desarrollo afectivo y social es importante entender que, en los 
niños de 0 a 6 años, ambos, están muy unidos $\mathrm{y}$ a veces es complicado distinguir lo que pertenece a uno y a otro. Esto se va a ir configurando en función de las variables o los factores internos de cada niño y de los externos, que modulan a los anteriores (Arce, 2015).

En esta etapa los niños son más sensibles a los pequeños acontecimientos, lo que les provoca cambios bruscos de humor $\mathrm{y}$ experimentan diferentes emociones, algunas muy cambiantes; predominan las emociones sobre los sentimientos, los estados emocionales son más intensos, pero al mismo tiempo más rápidos y menos profundos que los de los adultos (Soler, 2016).

De acuerdo con Papalia, Feldman y Martorell (2012) argumentaron que M. Lewis (2000), las emociones empiezan a desarrollarse durante la infancia, caracterizadas por las reacciones frente a los estímulos, siendo un elemento básico de la personalidad. Por consiguiente, el desarrollo emocional es un proceso ordenado que despliega una serie de emociones complejas a partir de otras más simples.

Durante los primeros años de vida, el niño aprenderá a manifestar sus propias emociones, a recibir las de los demás, y a responder ante ellas tratando de controlar las suyas propias. La manera como reaccione emocionalmente $\mathrm{y}$ cómo construya estos aprendizajes influirá en su bienestar y en su calidad de vida (Heras, 2016).

Por lo tanto, Muris (2014) y Stipek (1992) argumentan que "para experimentar emociones autoconscientes es necesario cierto desarrollo moral, siendo importante que los niños comprendan las reglas y normas que determinan cuáles son los comportamientos sociales que se consideran apropiados" (Tena, 2018, p. 50).

Dentro de este contexto, la confianza es una necesidad emocional que se expresa de forma racional y que nos permite relacionarnos con los demás y que, además, identifica nuestros valores. Pero, ¿a partir de qué se desarrolla esto? Según la primera etapa de desarrollo propuesta por Erikson "confianza frente a la desconfianza", depende del vínculo que haya establecido el bebé con su madre (Regader, 2018). Si predomina la confianza, los niños desarrollan la virtud de la esperanza, mientras que, si predomina la desconfianza, los niños verán al mundo como un lugar poco amistoso e impredecible (Papalia, Feldman y Martorell, 2012).

El permitir a un niño desde tempranas edades realizar sus propias elecciones en cuestiones sencillas pero trascendentales para él, como escoger qué ropa ponerse, o qué juguetes usar, o qué colores prefiere; es lograr que aprendan a gestionar sus emociones, su forma de actuar y descubrir pasiones (Valdez Ulloa, 2017).

Según Erikson, los niños al poder completar tareas por sí mismos con éxito, obtienen un sentido de independencia y autonomía, esto representa que poco a poco hagan frente a sus dudas $\mathrm{y}$ aprendan a resolver conflictos. Quienes completan esta etapa con gozo suelen contar con una autoestima sana y fuerte, mientras que los que no lo hacen suelen quedarse con una sensación de inestabilidad (Rodríguez, 2018).

En consecuencia, otro de los elementos básicos de la personalidad es la autoestima, constituyéndose en la conciencia de una persona de su propio valor. Dependiendo de su grado, se establecerá la calidad de relaciones sociales y afectivas, dando paso al sentimiento de felicidad. Cuando un niño construye una autoestima saludable se siente competente, seguro y valioso, al contrario, el niño con una baja autoestima no confiará en sus propias posibilidades ni en las de los demás (Guiainfantil, 2018). 
Sin embargo, la autoestima en los niños no se basa necesariamente en la realidad, más bien tienden a aceptar juicios de los adultos. Además, en la niñez temprana la autoestima tiende a ser dicotómica, es decir, de todo o nada "soy bueno" o "soy malo" (Papalia, Feldman, y Martorell, 2012).

La etapa de la iniciativa a la culpa se da en la edad del juego. Cuando el niño desarrolla su imaginación, comprende mejor y hace preguntas constantemente, lo que le proporciona un sentimiento de iniciativa que constituye el sentido de ambición y propósito (Zapata, y Alvear, 2015). Entonces, cuando los niños descubren sentimientos contradictorios, entre lo que quieren hacer y la aprobación social son capaces de autorregularse, perseguir propósitos y metas sin inhibirse demasiado por los sentimientos de culpa y el temor al castigo (Botempone Silva, Flores y Ramírez, 2012).

Muñoz, L. M. (2017) citó a Kopp (1982) quien argumentó que, “El proceso de desarrollo de la autorregulación comienza desde el nacimiento, siguiendo distintas fases, hasta lograr un nivel de autorregulación más flexible que le permite al infante enfrentar contextos cambiantes, alrededor de los tres años de edad" (p.809). Inicialmente guiada por los cuidadores principales que tiene el niño, quienes ocupan un rol central y, quienes además son los primeros referentes de vínculo afectivo fuerte que tiene el niño (Kim, y Page, 2013).

Haciendo referencia a la importancia que tiene el vínculo afectivo, resulta necesario enfatizar en el apego como agente transformador de la personalidad en las diferentes etapas evolutivas. Bowlby (1973), lo define como "cualquier forma de conducta que tiene como resultado el que una persona obtenga o retenga la proximidad de otro individuo diferenciado y preferido, que suele concebirse como más fuerte y/o más sabio" (p.122). A partir de la cual el bebé o el niño siente la seguridad necesaria para explorar y dominar su entorno, en las situaciones en las que no hay amenaza, la conducta del niño mayormente estará dedicada a la exploración de su medio externo. Cuando el niño se encuentra ante una situación amenazante buscará a su cuidador, quien es el proveedor de seguridad. La búsqueda de la proximidad, la base segura y el refugio seguro son los tres rasgos definitorios y las tres funciones, de una relación de apego (Dávila, 2015).

\section{La familia como principal agente de socialización}

El rol socializador de la familia está caracterizado por las funciones asistenciales, educativas y adaptativas, que contribuyen al desarrollo personal del ser humano, ésta función socializadora posee relevancia en la infancia, momento que requiere de protección, garantía de derechos, sostén emocional y social. Para Márquez, y Gaeta (2017) el contexto familiar es significativo en la atención, con mayor énfasis en los primeros años de vida, puesto que, la familia es la primera red de apoyo y escenario donde debe promoverse un ambiente sano para el desarrollo personal y social del individuo (Cardona, y otros, 2015). "Las interacciones sociales que se desarrollan en este espacio formativo, ayudan a los niños y niñas a comprenderse a sí mismos y a sus semejantes sociales como sujetos sociales e históricos, como productores de cultura; así, dan la oportunidad a la construcción de la base inicial para la vivencia efectiva de su ciudadanía" (Finco, 2015 p. 89).

El ser humano es socialmente activo, siempre que disponga de habilidades que le permitan enfrentarse a los desafíos cotidianos, para poder hacerlo es importante el rol protagónico de la familia, del adulto o 
cuidador primario, porque favorecen a nivel social y afectivo al individuo. Es función de la familia preparar a sus integrantes para ocupar roles sociales, control de impulsos, toma de decisiones, resolución de conflictos, cumplimiento de objetivos de desarrollo personal, fortalecimiento de valores, les permitirá convertirse en miembros sociales proactivos (Suárez y Vélez, 2018).

La familia funciona como un sistema biopsicosocial que tiene como objetivo responder a las demandas sociales, como un espacio propicio para la formación de la habilidad para relacionarse con otros, de brindar protección y afecto de tal manera que los padres están asignados al proceso de socialización (Simkin y Becerra, 2013). Por lo tanto, el papel de la familia en el desarrollo social se sustenta en la garantía de valores, acciones, crianza, afectividad, comunicación y educación, que son la base para el bienestar social del individuo.

El estilo parental se relaciona con la competencia emocional y social que se promueve en los hijos, ya que dichas variables están íntimamente relacionadas y son responsables de la adecuada evolución en el resto de ámbitos del desarrollo infantil (Ramírez, Ferrando y Sáenz, 2015). Por lo tanto, los contextos socioeducativos donde se desarrollan los niños se configuran como espacios y estructuras educativas que favorecen su personalidad (García, Gómez y Trillas, 2016). Trabajar en el desarrollo emocional siempre es un tema de gran utilidad que permite enriquecer a los padres de familia como testimonios de vida $y$ modelos que marcan una identidad personal y social en los hijos.

Paguay y Espinoza (2015, p. 22) destacan: "El contexto familiar es importante en la personalidad, y se desarrolla a raíz del proceso de socialización, en la que el niño asimila las actitudes, valores y costumbres de la sociedad". En este marco para determinar el accionar de los padres en el desarrollo emocional de los hijos, se lo evidencia en el aumento de la autoconciencia y equilibrio emocional. Así lo corrobora Melgosa (2017) al referirse sobre las emociones a través de un funcionamiento cíclico, es decir, que todas las emociones que el niño recibe son útiles para la supervivencia y el adulto está llamado a enseñar desde temprana edad a reconocer las mismas y las consecuencias que acarrea para la persona y los demás, cuando estas son negativas.

El rol fundamental que desempeñan los padres y madres a través de conductas parentales positivas procuran el bienestar de los niños y su desarrollo integral, Los niños y las niñas son competentes y capaces, pero también necesitan protección y orientación que les permita disfrutar de su infancia y posteriormente convertirse en adultos que se desenvuelvan asertivamente en la sociedad.

En el ambiente familiar se va conformando la conducta y el desarrollo de la personalidad en los niños, además, los padres ejercen una influencia poderosa en la vida emocional de los hijos, ya que se gestionan los primeros vínculos afectivos y sociales, los mismos que servirán en la relación personal y con sus pares. Este enunciado se sustenta con el aporte de Rangel y Valdés (2017, p. 105) al considerar que "La familia es la unidad fundamental y primaria donde el ser humano se va haciendo persona, es la primera institución donde descubre las formas básicas de la vida social y en la que aprende a relacionarse con los otros".

Como se viene mencionando, la formación juega un importante papel en el desarrollo de la personalidad del individuo como ser social y forma parte del proceso educativo; por tal razón, la educación se 
considera un sistema complejo de influencias en el que participa toda la sociedad, donde estas se asumen de manera muy particular y diferente por cada individuo. La familia como institución educativa debe integrarse a esta formación para que sus miembros se desarrollen en un ambiente armónico (Guevara, Herrera y García, 2014).

Es así que los agentes socializadores ejercen influencia a lo largo de los diferentes periodos evolutivos, por lo que se hace necesario un análisis que permita la comprensión de cada una de las etapas por las que atraviesa el individuo en su desarrollo. En síntesis, el rol de la familia como principal agente de socialización estará perennemente; puesto que, es el espacio en el que se establecen las primeras relaciones formadoras de la personalidad.

\section{CONCLUSIONES}

Las diferentes posiciones teóricas permiten tener una visión en retrospectiva sobre el inicio del estudio científico en relación a la conformación y estructura de la personalidad en la primera infancia, y más aún de las investigaciones y estudios que establecen las diferentes perspectivas conceptuales, teóricas, experimentales y prácticas en relación al desarrollo, características y evolución de las diferentes manifestaciones de la personalidad. En consecuencia, los investigadores pueden asumir diferentes posiciones de acuerdo a nuestra formación e intereses.

La personalidad en la primera infancia se encuentra conformada por conductas, comportamientos, emociones, pensamientos y rasgos de carácter que se irán consolidando a lo largo del ciclo vital. Es decir, no tiene fin y tampoco es generalizado, se plantea desde un enfoque de individualidad como respuesta a factores internos y externos. Cada niño presenta de acuerdo a su desarrollo socio afectivo características particulares en la formación de su personalidad, que lo distinguirá de los demás e influirá en aspectos relevantes como habilidades sociales, aprendizaje, entre otros.

Las emociones, la confianza, la autonomía, la autoestima y la autorregulación se constituyen como elementos básicos en el desarrollo de la personalidad, y específicamente del temperamento. Mismos que serán el resultado de las acciones socio educativas que reciban los niños de los adultos más cercanos que lo rodean. Su influencia favorece o no su desarrollo sano, en mayor o menor medida según los recursos personales que posean. De tal manera que, las conductas se irán consolidando en función de las reacciones o estímulos que la familia manifieste ante su comportamiento y de las diferentes experiencias que vaya acumulando.

\section{REFERENCIAS}

Aiken, L. (2003). Tests psicológicos y evaluación. México: Pearson Educación

Altajeros, F. (2004). Identidad, coexistencia y familia. Estudios sobre educación, 6, 105118

Arce, M. (2015). Crecimiento y desarrollo infantil temprano. Revista peruana de Medicina Experimental y Salud Pública, 574-578. Obtenido de https://www.redalyc.org/pdf/363/36342 789023.pdf

Botempone Silva, L., Flores M., R.d., y Ramírez R., L.N. (2012). La construcción de la identidad personal y el desarrollo de la auto-autoría. El Ágora, 421-436. Obtenido de

https://revistas.usb.edu.co/index.php/Ag ora/article/view/83/53

Bowlby, J. (1973). Separation: Anxiety y Anger. Attachment and Loss (vol. 2); (International psychoanalytical library № 95). London: Ho-glby, J. (1985). La separación afectiva. Barce-lona: Paidós. pp 292 
Caballo, V., Salazar, I., e Irurtria, M. (2011). Trastornos de la Personalidad. En V. Caballo, I. Salazar y J. Carrobles (Dirs.), Manual de Psicopatología y Trastornos Psicológicos. (pp.397-422). Madrid: Pirámide

Cardona, Á., Valencia, E., Duque, J., LondoñoVásquez, D. (2015). Construcción de los planes de vida de los jóvenes: una experiencia de investigación en la vereda La Doctora, Sabaneta (Antioquia). Aletheia. Revista de Desarrollo Humano, Educativo y Social Contemporáneo. Obtenido de http://www.scielo.org.co/pdf/aleth/v7n2 /v7n2a05.pdf

Carrillo Siles, B. (2009). La personalidad Infantil. Revista Digital Innovación y Experiencia Educativa, (15), 1-8. Obtenido de

https://archivos.csif.es/archivos/andaluci a/ensenanza/revistas/csicsif/revista/pdf /Numero_15/BEATRIZ_CARRILLO_1.pdf

Craig, G. J. (2009). Desarrollo Psicológico. México: Pearson Educación, p 167

Dávila, Y. P. (2015). La influencia de la familia en el desarrollo del apego. Revista de la Universidad de Cuenca, 121-130. Obtenido de http://dspace.ucuenca.edu.ec/bitstream/ 123456789/22943/1/10.pdf

Engel, B. (1996). Teorías de la personalidad. México: Mc Graw Hill

Finco, D. (2015). Igualdad de género en las instituciones educativas de la primera infancia brasileña. Revista Latinoamericana de Ciencias Sociales, Niñez y Juventud, 13 (1), 85-96. Obtenido de

http://www.scielo.org.co/pdf/rlcs/v13n1 /v13n1a04.pdf

García, T. M., Gómez, M., \& Trilla, J. (2016). Participación infantil y construcción de la identidad del sujeto. Búsqueda, 3(16), 126-127. Obtenido de https://revistas.cecar.edu.co/index.php/B usqueda/article/view/206

González, G. A. (2013). Teorías de la personalidad (Primera ed.). México: Red
Tercer Milenio. Obtenido de http://www.aliat.org.mx/BibliotecasDigit ales/Psicologia/Teorias_de_la_personalida d.pdf

Guevara F., G. E., Herrera R, J, y García P., Y. (2014). La integración de las influencias educativas de la universidad y la familia en la formación del profesional de la educación superior. Gaceta Médica Espirituana, 16(2), 131-141. Recuperado en 22 de noviembre de 2020, de http://scielo.sld.cu/pdf/gme/v16n2/gme 14214.pdf

Guiainfantil. (2018). La autoestima y los niños. ¿Cómo podemos los padres ayudar a nuestros hijos a que tengan una buena autoestima? Disponible en: https://www.guiainfantil.com/salud/auto estima/index.htm (Consultado 22 de noviembre de 2020)

Guirao-Goris, J. A., Olmedo S., A., y Ferrer F., E. (2008). El artículo de revisión. Revista iberoamericana de enfermería comunitaria, 1(1), 1-25. Obtenido de https://www.uv.es/joguigo/valencia/Rec erca_files/el_articulo_de_revision.pdf

Heras, D., Cepa, A., y Fernando, L. (2016). Desarrollo emocional en la infancia. Un estudio sobre las competencias. INFAD Revista de Psicología. Obtenido de http://www.infad.eu/RevistaINFAD/OJS/i ndex.php/IJODAEP/article/view/217

Kim, H. y Page, T. (2013). Emotional Bonds with Parents, Emotion Regulation, and School-Related Behavior Problems Among Elementary School Truants. Journal of Child Family Studies22, pp. 869-878. Doi:10.1007/s10826-012-9646-5

Liberati, A., Altman, D. G., Tetzlaff, J., Mulrow, C., Gotzsche, P. C., Ioannidis, J. P. A., \& Moher, D. (2009). The PRISMA statement for reporting systematic reviews and metaanalyses of studies that evaluate healthcare interventions: Explanation and elaboration. British Medical Journal, 339, b2700. Doi: https://doi.org/10.1136/bmj.b2700

Linares, A. (2009). Desarrollo cognitivo: las teorías de Piaget y de Vigotsky. Obtenido de 
http://www.paidopsiquiatria.cat/files/Te orias_desarrollo_cognitivo.pdf

Márquez, M. y Gaeta, M. (2017). Desarrollo de competencias emocionales en preadolescentes: el papel de padres y docentes. Revista Electrónica Interuniversitaria de Formación del Profesorado, 20(2), 221-235. DOI: https://doi.org/10.6018/reifop/20.2.232 941

McCrae, R. R., y Costa, P. T. (2012). Personality in adulthood (2nd ed.). New York: The Guilford Press

Melgosa, J. (2017). Preservando la salud mental. Revista de Investigación Apuntes Universitarios, 7(2), 84-89. Obtenido de https://www.redalyc.org/pdf/4676/4676 52767009.pdf

Montaño S., M., Palacios Cruz, J., y Gantiva, C. (2009). Teorías de la personalidad. Un análisis histórico y su medición. Avances de la displina, 3(2), 81-107. Obtenido de Teorías de la personalidad. Un análisis histórico del concepto y su medición, en https://www.redalyc.org/pdf/2972/2972 25531007.pdf

Morán, M. C., Fínez, M. J., y Fernández-Abascal, E. G. (2017). Sobre la felicidad y su relación con tipos y rasgos de personalidad. Clínica y salud, 28(2), 5963.

https://reader.elsevier.com/reader/sd/pi i/S1130527416300603?token=60345D86 2455B209BE3D3E8956AC2947C448CC34 53C6593AA6BCE8FCB6E38390688F6817 EB7EEF333413FB33B0107823

Muñoz, L. M. (2017). La autorregulación y su relación con el apego en la niñez. Revista Latinoamericana de Ciencias Sociales, Niñez y Juventud, 15(2), 807-821. Obtenido de https://www.redalyc.org/pdf/773/77352 074003.pdf

Muris, P. M. (2014). Bound to feel bad about oneself: Relations between attachment and the self-conscious emotions of guilt and shame in children and adolescents. Journal of Child and Family Studies

Paguay Gañay, R. L., y Espinoza Vera, M. M. (2015). El Ambiente familiar y su influencia en la seguridad emocional y afectiva (Bachelor's thesis). Obtenido de http://repositorio.unemi.edu.ec/bitstrea $\mathrm{m} / 123456789 / 2404 / 1 /$ EL\%20AMBIENT E\%20FAMILIAR\%20Y\%20SU\%20INFLUE NCIA\%20EN\%20LA\%20SEGURIDAD\%20 EMOCIONAL\%20Y\%20AFECTIVA.pdf

Papalia, D., Feldman, R., y Martorell, G. (2012). Desarrollo humano (desarrollo humano ed.). (J. F. María Elena Ortiz Salinas, Trad.) México: McGRAWHILL/INTERAMERICANA EDITORES, S.A. DE C.V. Obtenido de https://www.academia.edu/20423118/D esarrollo-humano-Papalia-12a p.174

Pervin, L y John, O. (1998). Personalidad: teoría e investigación. México: Manual Moderno

Quezada, P. (2018). El entorno social y el aprendizaje. Obtenido de https://www.researchgate.net/publicatio n/327403136_El_entorno_social_y_el_apre ndizaje

Ramírez, A.; Ferrando, M. y Sainz A. (2015). ¿Influyen los estilos parentales y la inteligencia emocional de los padres en el desarrollo emocional de los hijos escolarizados en $2^{\circ}$ ciclo de educación infantil? Revista Acción Psicológica, 12(1), 65-78. Obtenido de https://www.redalyc.org/pdf/3440/3440 41426007.pdf

Rangel, Y. R., y Valdés, M. D. L. Á. G. (2017). Un acercamiento a la función educativa de la familia. Revista Cubana de Medicina General Integral, 33(1), 100-114.

Regader, B. (2018). La teoría del desarrollo psicosocial de Erik Erikson

Rodríguez, E. (17 de diciembre de 2018). La mente es maravillosa. Obtenido de Las etapas del desarrollo psicosocial de Erikson: https://lamenteesmaravillosa.com/lasetapas-del-desarrollo-psicosocial-deerikson/

Romero, E. L.-F. (2002). La estructura de los rasgos de personalidad en adolescentes: El Modelo de Cinco Factores y los Cinco Alternativos [The structure of personality traits in adolescents: The Five-Factor 
Model and the alternative five]. Psicothema 14(1), 134-143. Obtenido de http://www.psicothema.com/pdf/697.pd $\mathrm{f}$

Seelbach, G.A. (2013). Teorías de la personalidad, México, Red Tercer Milenio, 2013. http://www.aliat.org.mx/BibliotecasDigit ales/Psicologia/Teorias_de_la_personalida d.pdf

Simkin, H., y Azzollini, S. (2015). Personalidad, Autoestima y Espiritualidad-Religiosidad desde el Modelo y la Teoría de los Cinco Factores. PSIENCIA: Revista Latinoamericana de Ciencia Psicológica, 7(2)339-361. http://www.psiencia.org/psiencia/7/2/P SIENCIA_Revista-Latinoamericana-deCiencia-Psicol\%C3\%B3gica_72_Simkin_Azzollini.pdf

Simkin, H., Becerra, G. (2013). El proceso de socialización. Apuntes para su exploración en el campo psicosocial. Ciencia, docencia y tecnología, 24 (47):119-142. Disponible en

http://www.redalyc.org/pdf/145/14529 884005.pdf

Soler, V. (2016). Desarrollo socioafectivo. España: Editorial Síntesis S.A. Obtenido de https://www.sintesis.com/data/indices/9 788490773055.pdf

Stipek, D. R. (1992). Self-evaluation in young children. Monographs of the Society for Research in Child Development

Suárez, P. y Vélez, M. (2018). El papel de la familia en el desarrollo social del niño: una mirada desde la afectividad, la comunicación familiar y estilos de educación parental. PSICOESPACIOS, 12(20), 173-198. Obtenido de http://revistas.iue.edu.co/revistasiue/ind ex.php/Psicoespacios/article/view/1046/ 1315

Tena, E. L. (2018). Las emociones autoconscientes en niños y niñas españoles /as de 7 a 11 años. (Tesis doctoral). Universidad Complutence de madrid, Madrid. Obtenido de https://eprints.ucm.es/46730/1/T39652. pdf

Tintaya, C. (2019). Psicología y Personalidad. Revista de Psicología vol. no 21. Bolivia. La Paz. Obtenido de http://www.scielo.org.bo/pdf/rip/n21/n 21_a09.pdf

Valdez Ulloa, D.K. (2017). La autonomía y la estimulación de la confianza en el niño y niña de 5 a 6 años (examen complexivo). UTMACH, Unidad Académica de Ciencias Sociales, Machala, Ecuador. Obtenido de http://repositorio.utmachala.edu.ec/bitst ream/48000/11415/1/E-

7385_VALDEZ\%20ULLOA\%20DIANA\%20 KATHERINE.pdf

Zapata, A. R., y Alvear, S. G. (2015). Guía didáctica lúdica para incentivar el desarrollo intregral de los niños y niñas de 12 a 36 meses del centro infantil del buen vivir "semillas de miraflores alto" del cantón Cayambe. Tesis de licenciatura. Universidad Politécnica Salesiana de Quito, Quito. Obtenido de https://dspace.ups.edu.ec/bitstream/123 456789/7743/1/UPS-QT06527.pdf 